\title{
Cáncer de la vesícula biliar. Manejo de pacientes con invasión de la túnica muscular
}

\author{
Xabier de Aretxabala U 1,2 , Iván Roa $\mathrm{E}^{3}$, Javier Mora Pa, \\ O scar Pincheira $S^{a}$, Luis Burgos $S^{1,2}$, Jorge Silva $A^{1}$, \\ Juan Carlos Roa $\mathbf{S}^{3}$.

\section{Management of gallbladder cancer with invasion of the muscular layer}

Background: Gallbladder cancer is the leading cause of death of cancer among women in Chile. Detection of early forms of the disease during the pathological study of the cholecystectomy specimen is common. The management of these cases, specially those with invasion of the muscular layer is under discussion. Aim: To analyze the clinical features of patients with gallbladder cancer and muscular layer invasion and their prognosis after a simple cholecystectomy. Patients and methods: We studied a series of 46 patients with gallbladder cancer and invasion of the muscular layer. In the majority of cases diagnosis was performed at the moment of specimen study. Eleven patients underwent reoperation. Of these, ten underwent lymphadenectomy and liver resection while one patient underwent resection of the cystic duct stump. Results: During follow up, five patients died as a consequence of gallbladder cancer. Overall survival was $78 \%$, with no differences between patients subjected or not a new operation. Conclusions: Gallbladder cancer with muscular infiltration has a good prognosis, independent of the type of therapy (Rev Méd Chile 2004; 132: 183-8).

(Key Words: Cholecystectomy; Gallbladder diseases; Gallbladder neoplasms)

Recibido el 8 de abril, 2003. Aceptado en versión corregida el 1 de diciembre, 2003.

Financiado: Proyecto FONDECYT \#1010523.

${ }^{1}$ Departamento de Cirugía, Universidad de La Frontera. ${ }^{2}$ Clínica Alemana de Temuco.

${ }^{3}$ Unidad de Anatomía Patológica, Universidad de La Frontera.

anterno de Medicina

L a problemática del cáncer de la vesícula biliar _en Chile, determina que en muchas ocasiones debamos resolver problemas relacionados a su manejo basándonos en nuestra experiencia local. Esto, debido principalmente a la baja incidencia

Correspondencia a: Xabier de Aretxabala U. Departamento de Cirugía, Universidad de la Frontera. Fax: 45-325761. E mail: aretxaba@ufro.cl que este tumor posee en países desarrollados, de los cuales frecuentemente obtenemos la información para el manejo de la mayoría de las enfermedades oncológicas ${ }^{1-4}$.

Debido a nuestra alta incidencia de cáncer de vesícula biliar, nos vemos enfrentados al manejo de pacientes con lesiones tempranas, en los que no existe consenso sobre el tratamiento más adecuado de ellos. En general, los pacientes con tumores tempranos son aquellos en los que el 
tumor está circunscrito a la mucosa o a la túnica muscular.

Dentro de estos pacientes, aquellos con invasión de la túnica mucosa representan un particular grupo de pacientes, asociados a un muy buen pronóstico, en los que la simple colecistectomía ha sido considerada como la terapia de elección ${ }^{3-6}$.

En los pacientes con invasión de la túnica muscular la situación no es tan clara, existiendo incluso opiniones que este tipo de tumores no debiera considerarse como un tumor temprano. Con el objeto de evaluar el pronóstico de estos pacientes y los posibles factores asociados a él, analizamos las características clínicas en una serie de ellos con especial énfasis en el pronóstico de ellos luego de la colecistectomía simple.

\section{Material y MÉtodo}

Desde 1989 hasta la fecha, todos los pacientes portadores de un tumor catalogado como potencialmente curativo, diagnosticado en la Unidad de Anatomía Patológica de la Universidad de La Frontera han sido registrados de manera prospectiva. Un tumor potencialmente curativo es definido como aquel en que el tumor es extirpado completamente al momento de la colecistectomía inicial o que la presencia de tumor está ubicada en áreas potencialmente resecables. Todos los pacientes fueron evaluados por uno de los autores del manuscrito (XA) y catalogados como potencialmente curativos basándose en el protocolo operatorio, comunicación con el cirujano que realizó la primera intervención y de la biopsia de la pieza de colecistectomía. Durante el período de estudio, un total de 316 pacientes han sido ingresados en este protocolo, de los cuales, un total de $46(14,5 \%)$ pacientes tuvieron cáncer de la vesícula biliar con infiltración de la túnica muscular ( $\mathrm{p} \mathrm{t1b}$ ). El seguimiento de los pacientes varió entre 2 y 131 meses (promedio 45, mediana 36, DS 39,6). Fueron evaluados los siguientes factores a los pacientes: sexo, edad, intervención realizada, diagnóstico preoperatorio, diagnóstico postoperatorio, infiltración de la pared de la vesícula, tipo macroscópico y evolución. Los pacientes fueron clasificados conforme a la Unión Internacional contra el Cáncer ${ }^{7}$. En un comienzo, los pacientes fueron manejados conforme a nuestro protocolo de manejo de pacientes portadores de un tumor potencialmente curativo, el que consiste básicamente en la realización de una reintervención posterior al diagnóstico realizado durante el estudio de la pieza de colecistectomía. Esta reintervención se efectúa con la finalidad de realizar una linfadenectomía del pedículo hepático y la resección de los segmentos IV y V. Motivados por el buen pronóstico general de los pacientes con invasión muscular, se resolvió considerar a la colecistectomía como terapéutica y no continuar con la ejecución de reintervenciones.

Las curvas de sobrevida fueron construidas de acuerdo al método de Kaplan y Meier y las diferencias entre las series analizadas de acuerdo al long rank test.

\section{RESUlTADOs}

La serie estuvo compuesta por 9 pacientes de sexo masculino y 37 de sexo femenino. La edad de los pacientes fluctuó entre 30 y 85 años con un promedio de 57 años. Trece (28\%) pacientes tuvieron una edad menor a 45 años.

En la mayoría de los pacientes, el diagnóstico preoperatorio fue de patología benigna, destacando que 20 de los pacientes tuvieron un diagnóstico de colecistitis aguda. En sólo dos pacientes se sospechó la existencia de un cáncer de vesícula biliar, siendo este diagnóstico planteado durante el preoperatorio (Tabla 1). Uno de estos pacientes presentaba una masa de barro biliar que hizo sospechar la existencia de un tumor en el interior de la vesícula. Sin embargo, este hecho fue coincidente, ya que el diagnóstico de tumor fue

Tabla 1. D iagnóstico pre y post operatorio

\begin{tabular}{|lcc|}
\hline & Preoperatorio & Postoperatorio \\
\hline Colecistitis crónica & 18 & 21 \\
Colecistitis aguda & 20 & 21 \\
Cáncer vesícula & 2 & 1 \\
Cole + coledocolitiasis & 2 & 1 \\
Pólipo & & 1 \\
Otro & 4 & 1 \\
\hline
\end{tabular}


realizado luego del examen histológico de la pieza. El restante paciente efectivamente poseía una lesión vegetante detectada por la ecotomografía que se confirmó como lesión tumoral.

Veinte pacientes fueron intervenidos de urgencia, generalmente por el diagnóstico de colecistitis aguda. El diagnóstico postoperatorio mantuvo igual tendencia diagnosticándose una colecistitis aguda en 21 pacientes. De igual modo, sólo en un paciente se sospechó la existencia de un cáncer de la vesícula biliar, y correspondía a aquel en que el tumor había sido sospechado en el preoperatorio.

De los 39 pacientes en los que se logró consignar el tipo macroscópico de la lesión, en 24 ésta no fue identificada durante el examen de la pieza macroscópica posterior a la fijación, siendo sólo identificada luego del estudio histológico. En 14 pacientes, fue detectada una lesión elevada, mientras que en un caso el tumor poseía la apariencia de una úlcera.

En sólo un paciente el procedimiento fue efectuado vía laparoscópica, siendo todos los restantes ejecutados vía abierta. La totalidad de los pacientes estudiados presentaban una colelitiasis concomitante.
Once pacientes fueron reintervenidos posterior a la colecistectomía, en diez la intervención fue con la finalidad de efectuar una linfadenectomía y la resección del lecho de la vesícula biliar. De estos pacientes, en sólo uno se encontró compromiso tumoral en la zona resecada. Esta paciente presentaba un nódulo metastásico a nivel del tejido hepático resecado. La restante paciente reoperada fue debido a la existencia de tumor a nivel del borde de sección del conducto cístico, lo que motivó la reintervención con la finalidad de resecar la porción distal del conducto y lograr un borde de sección libre de enfermedad tumoral. La sobrevida global de la serie estudiada fue de $78 \%$, no existiendo diferencias cuando se compararon el grupo sometido a reintervención con aquel sometido a simple colecistectomía (Figuras 1 y 2).

De la serie de pacientes, 6 pacientes han fallecido; el seguimiento de los pacientes fallecidos fue de 11, 14, 20, 27, 34 y 51 meses respectivamente. De los pacientes fallecidos, dos habían sido sometidas a una linfadenectomía y a la resección en cuña del hígado. Ambos pacientes habían sido intervenidos con ánimo curativo. En la Tabla 2 se presentan las características clínicas de los pacientes fallecidos.

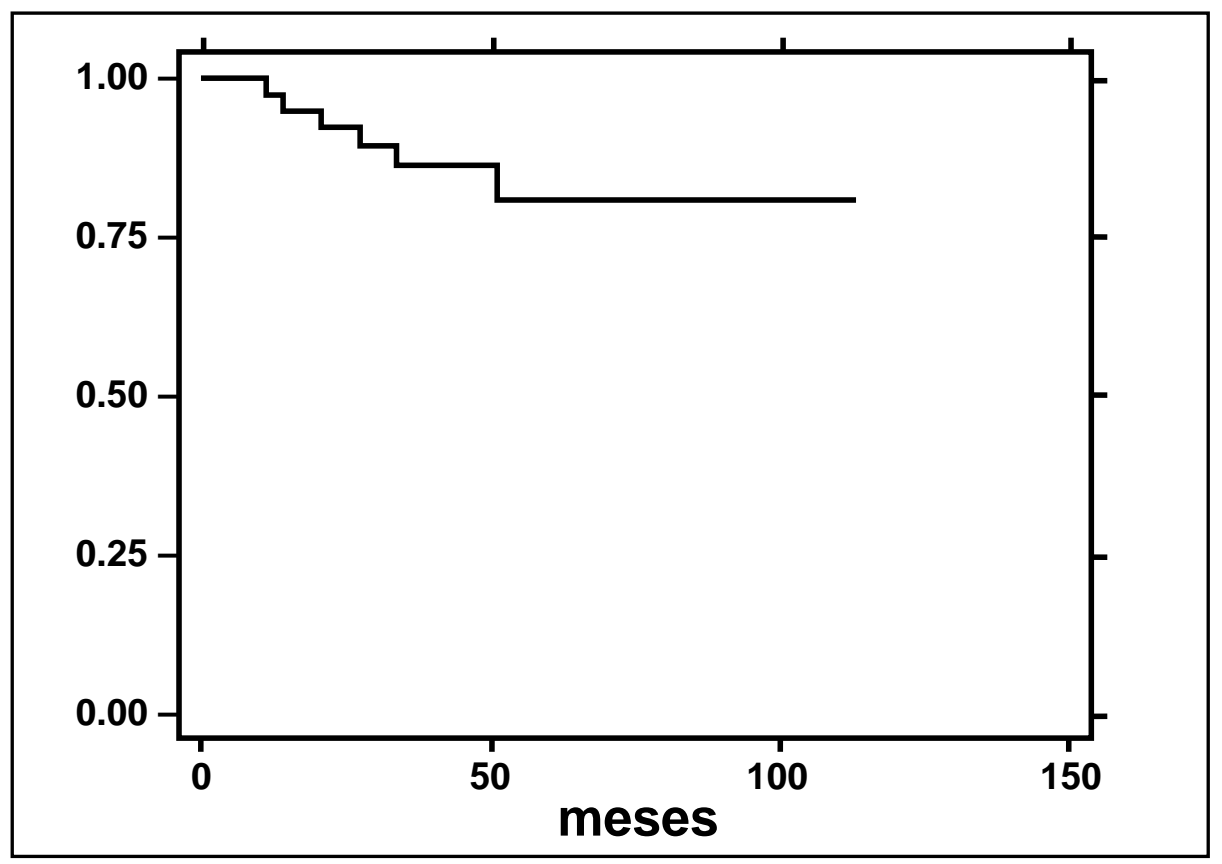

Figura 1. Curva de sobrevida global de la totalidad de los pacientes portadores de un tumor muscular. 


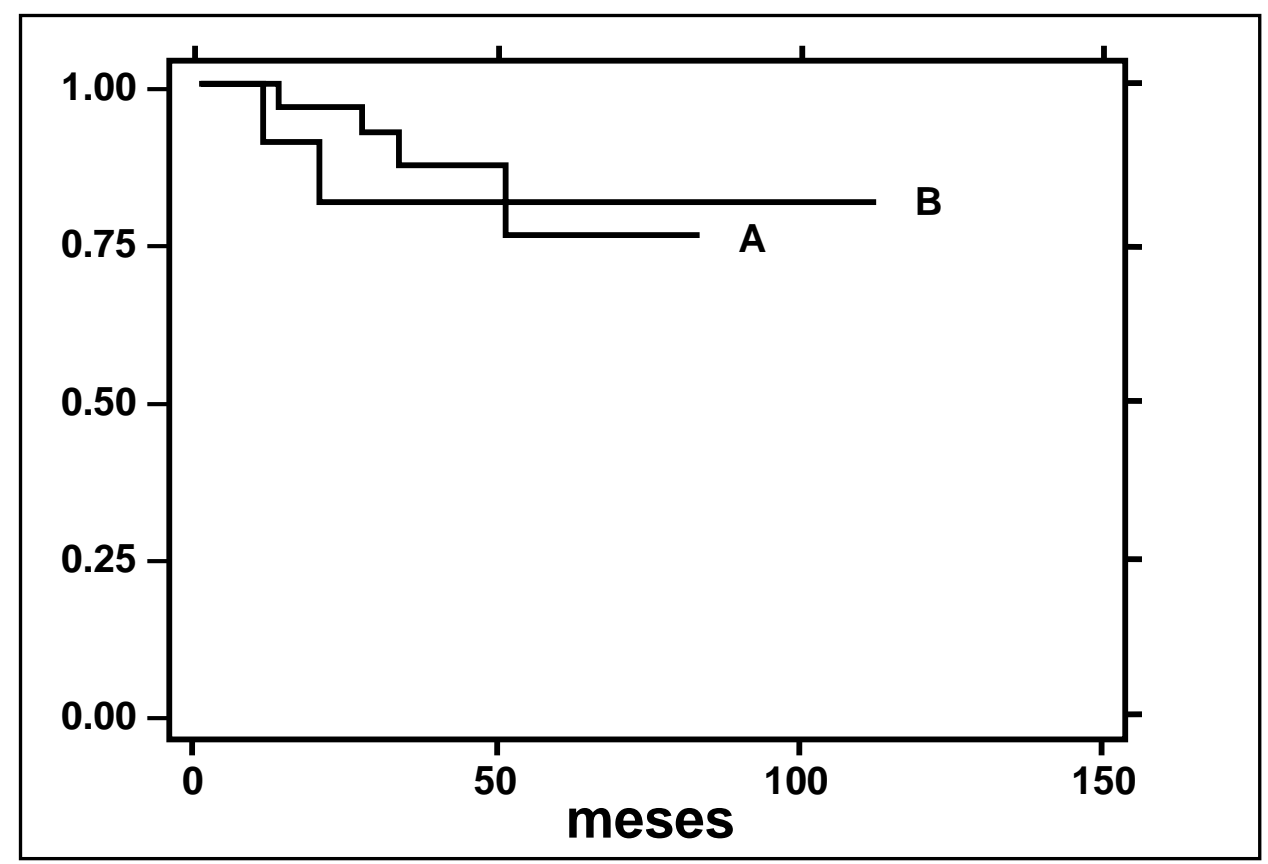

Figura 2. a) Pacientes no sometidos a reintervención. b) Pacientes sometidos a reintervención $\mathrm{p}>0,05$.

Tabla 2. Características pacientes fallecidos

\begin{tabular}{|c|c|c|c|c|c|c|}
\hline \multicolumn{4}{|c|}{ Edad } & \multicolumn{2}{|c|}{ Histología } & Sobrevida \\
\hline $\mathrm{JC}$ & 57 años & $\mathrm{L}(-)$ & $\mathrm{H}(-)$ & Mod Dif & Protruido & 20 meses \\
\hline TB & 41 años & $\mathrm{L}(-)$ & $\mathrm{H}(+)$ & Mod Dif & Plano & 11 meses \\
\hline $\mathrm{BN}$ & 43 años & & & Pobre & Plano & 51 meses \\
\hline $\mathrm{RP}$ & 70 años & & & Bien & Plano & 34 meses \\
\hline EA & 75 años & & & Mod & Plano & 14 meses \\
\hline NR & 47 años & & & Mod & Protruido & 27 meses \\
\hline
\end{tabular}

\section{Discusión}

El cáncer de la vesícula biliar constituye en la actualidad una enfermedad común a la que nos vemos enfrentados diariamente. A pesar del desarrollo de nuevas técnicas de imágenes, el diagnóstico de lesiones tempranas de la vesícula biliar persiste siendo un hallazgo posterior al estudio histológico de la pieza de colecistectomía. Sólo algunas series japonesas muestran un mayor por- centaje de diagnóstico preoperatorio, sin embargo es posible que la mayor incidencia de lesiones tumorales protruidas, sin coexistencia con litiasis, pueda ser responsable de esta diferencia. En este sentido, las series mencionadas anteriormente, muestran una coexistencia de litiasis entre 53 y $72 \%$, lo que se compara con la presente serie en la que la totalidad de los pacientes tenían coexistencia de litiasis ${ }^{8,9}$. Estas características observadas en la población japonesa, permiten que la ecotomo- 
grafía logre sospechar el diagnóstico en estos pacientes.

Una de las mayores controversias la constituyen el manejo de los pacientes con tumores pequeños detectados posterior a la colecistectomía. En general, cuando la invasión está limitada a la túnica mucosa, la colecistectomía simple es considerada el tratamiento de elección, a pesar de la descripción de pacientes que presentan recurrencias en este estado $1,3,10-12$. Estos pacientes presentan sobrevidas que superan el $90 \%$ a 5 años. En el otro extremo, se encuentran los pacientes con invasión de la túnica subserosa. Este grupo de pacientes, poseen una sobrevida cercana a $40 \%$, existiendo prácticamente consenso entre los diferentes centros en que la colecistectomía simple sería insuficiente como tratamiento $6,10,13$. El grupo de pacientes estudiado constituye un grupo de real interés debido a que su sobrevida con la colecistectomía simple es elevada y, por ende, cabe poco espacio para que un procedimiento quirúrgico pudiese mejorar su sobrevida. Debido a que en un comienzo, nuestra conducta era preferentemente de tipo resectiva, efectuábamos la reintervención de estos pacientes con la finalidad de realizar una linfadenectomía y la resección del lecho de la vesícula biliar. Los resultados de estas reintervenciones, aun cuando su número era escaso no mostraban evidencias de su utilidad. La revisión de la bibliografía extranjera es controversial a este respecto, Ouchi, por ejemplo, reportó la presencia de recurrencias en tres de cinco pacientes con una enfermedad tumoral limitada a la túnica muscular ${ }^{14}$. Estos resultados contrastan con los observados en nuestro grupo.

\section{REFERENCIAS}

1. De Aretxabala X, Roa I, Burgos L, araya JC, FonsECA L. Gallblader Cancer in Chile. A report on 54 potentially resectable tumors. Cancer 1992; 69: 60-5.

2. SERRA I. ¿Ha disminuido la mortalidad por cáncer de la vesícula biliar en Chile? Rev Méd Chile 2001; 129: 1079-84.
La colecistectomía laparoscópica constituye el tratamiento de elección de la patología litiásica, sin embargo llama la atención que en esta serie de pacientes sólo una paciente fue sometida a una colecistectomía laparoscópica. Este hecho, posiblemente muestre la realidad quirúrgica en instituciones públicas chilenas, en las cuales aún el método laparoscópico no está completamente masificado especialmente en cirugía de urgencia. Es importante insistir en la necesidad de extremar los cuidados de ruptura vesicular durante el procedimiento, debido a la posible existencia de un tumor concomitante y por lo tanto de diseminación peritoneal. Datos aún no publicados de nuestro centro, muestran que las recurrencias en pacientes con enfermedad T2, son mayores en pacientes operados vía laparoscópica comparado a aquellos operados vía abierta. De igual modo, Z'Aggren mostró cómo la sobrevida es influenciada por la existencia de ruptura vesicular en los pacientes operados vía laparoscópica ${ }^{15}$.

Como conclusión de este trabajo podemos decir que el cáncer de la vesícula con infiltración de la túnica muscular posee un buen pronóstico, incluso luego de la simple colecistectomía. Es difícil validar a la colecistectomía extendida como método terapéutico en este particular grupo de pacientes, más aún, cuando la sola colecistectomía posee sobrevidas elevadas. El análisis de los pacientes fallecidos tampoco permite obtener un patrón característico que se asocie a un pobre pronóstico y, posiblemente, se requiera de una mayor serie de pacientes con compromiso muscular fallecidos que permita evaluar características propias de estos pacientes.

3. Benoist S, Panis Y, Fagniez PL. Long term results after curative resection for carcinoma of the gallbladder. Am J Surg 1998; 175: 119-22.

4. Donohue JH, Nagorney DM, Grant CS, Tsushima K, ILSTRUP DM, ADSON MA. Carcinoma of the gallbladder: does radical resection improve the outcome? Arch Surg 1990; 125: 237-41.

5. Shirai $Y$, Yoshida K, Tsukada K, Muto T, Watanabe $\mathrm{H}$. Radical resection for gallbladder carcinoma: Long term results. Ann Surg 1992; 16: 565-8. 
6. de Aretxabala X, Roa I, Burgos L, Araya JC, VILASECA M, SiLVA J. Curative resection in potentially respectable tumors of the gallbladder. Eur J Surg 1997; 163: 419-26.

7. Hermanek P, Hutter RVP, Sobin LH, Wagner G, WitTeKIND CH. UICC TNM atlas. Fourth edition. Berlin Heidelberg New York. Springer-Verlag. 1997.

8. Onoyama H, Yamamoto M, TaKada M, URakana T, AJIKI T, YAMADA I ET AL. Diagnostic imaging of early gallbladder cancer. Retrospective study of 53 cases. World J Surg 1999; 23: 708-12.

9. Yamaguchi $K$, Chijina $K$, Saiki S, Nishihara $K$, TAKASHIMA M, KaWAKAMI T et aL. Retrospective analysis of 70 operations for gallbladder carcinoma. Br J Surg 1997; 84: 200-4.

10. Shirai Y, Yoshida K, Tsukada K, Muto Y. Unapparent carcinoma of the gallbladder. An appraisal of a radical second operation after simple cholecystectomy. Ann Surg 1992; 215: 326-31.
11. Wagholkar GD, Behari A, Krishnan N, Kumar A, SiKora A, SAXENA R ET AL. Early Gallbladder Cancer. J Am Coll Surg 2002; 194: 137-41.

12. Shiari Y, Yoshida K, Tsukada K, Muto T, Watanabe $\mathrm{H}$. Early carcinoma of the gallbladder. Eur J Surg 1992; 158: 545-8.

13. Barlett DL, Fong Y, Fortner JG, Brennan MF, BLUMGART P. Long term results after resection for gallbladder cancer, implications for surgical management. Ann Surg 1996; 224: 639-46.

14. Ouchi K, Sugawara T, Ono H, Fujita T, Kamiyama $Y$, KaKUgaWa Y ET aL. Diagnostic capability and rational resectional surgery for early gallbladder cancer. Hepatogastroenterology 1999; 46: 1557-60.

15. Z'Aggren K, Birrer S, Maurer CA, Wehrl H, Kiaiber C, BAER HU. Incidence of port site recurrence after laparoscopic cholecystectomy for preoperatively unsuspected gallbladder carcinoma. Surgery 1998; 124: 831-8. 